\title{
Effect of supervised versus self-directed exercise and dietary practices on body weight and glycaemic control with individuals having type 2 diabetes
}

\author{
๑ Alemmebrat Kiflu Adane \\ Department of Sport Science, College of Natural and Computational Sciences, Addis Ababa University, Ethiopia.
}

\begin{abstract}
Similar to a highly developed country, these days the rapid and parallel increase in sedentary lifestyle and type 2 diabetes (T2DM) is becoming one of the major public health concerns of Ethiopia. Despite the fact that pharmacological medicines are mostly ordered by physicians as a treatment modality to T2DM, regular physical exercise and healthy diet habits are not well understood and practiced throughout society. Previous studies also documented that the latter two approaches are the two key management tools for diabetes and it assists in preventing diabetes-related health complications, insulin resistance, and T2DM. The present study examined the difference in body weight and fasting plasma glucose (FPG) improvement in 16 moderately overweight male individuals having T2DM for 10wks intervention, to this end experimental with retrospective cohort study design was used. After qualified the recruitment criteria subjects were randomly categorized into two groups, i.e. experimental $(\mathrm{n}=8)$ and control $(\mathrm{n}=8)$. Individuals who have received a supervised exercise program and close dietary advice were compared with those who were engaged in self-directed exercise and also having their own eating habits over the study program. To analysis, the data, dependent t-test and Pearson's correlation were used. In all cases, the statistical significance threshold was $\mathrm{P}<0.05$. At the end of the intervention, significant bodyweight reduction and FPG improvement were observed in the experimental group as compared to their counterparts. Therefore, it was concluded that professionally supervised regular exercise with a close dietary practice should be the main choice of individuals having $\mathrm{T} 2 \mathrm{DM}$ in order to manage and achieve
\end{abstract}

sustainable body weight control and FPG for better health and treatment modalities.

Keywords. Body weight, glycaemic control, selfdirected exercise, type II diabetes.

\section{Introduction}

Bodyweight is of critical importance for promoting overall good health and optimizing performance. Although many individuals are able to regulate and control their body weight with relative ease, there are countless others who struggle with trying to prevent excess weight gain, achieve significant weight loss. Understanding and achieving the desired body weight is essential. However, there are numerous genetic, environmental, physiologic, psychological, and behavioral factors that could potentially influence energy intake or energy expenditure, causing the energy balance equation to become unbalanced and lead to weight gain or weight loss (Potteiger, 2018).

Diabetes mellitus (DM) is a major public health issue affecting populations around the world. It is a group of metabolic diseases characterized by an elevated blood glucose concentration (i.e., hyperglycemia) as a result of defects in insulin secretion and/or an inability to use insulin (Ehrman et al., 2018; Shaw et al., 2010). One of the major driving forces behind the diabetes epidemic is the escalating obesity epidemic. Obesity rates are ever- 
increasing due to a sedentary lifestyle and caloric excess. Increasing body mass index (BMI) is associated with an increased prevalence of diabetes in all ethnic groups. Increased visceral adiposity is associated with insulin resistance, and insulin resistance is known to be an important factor underlying the development of T2DM (Gallagher \& LeRoith, 2012).

Studies have revealed that worldwide, the incidence of type 2 diabetes has jumped 50 percent in the last 10 years and the number of people with type 2 diabetes is predicted to be more than 190 million people, and some experts predict that if the current trends continue that figure could surge to over 300 million by the year 2025 (Metzger, 2006). The prevalence of type 2 diabetes in African countries ranged from $1 \%$ in rural Uganda to $12 \%$ in urban Kenya (Hall et al., 2011).

In Ethiopia, since the last decades, there is a rapid and large increase in the number of overweight and obese individuals so that more individuals are becoming overweight or obese than ever before. In this connection, the prevalence and incidence of type-2 diabetes have been surveyed. For example, a systematic review of Abebe et al. (2017) indicated that currently Ethiopia has been challenged by the growing magnitude of noncommunicable diseases (NCDs) such as diabetes. Ethiopia is among the top four countries with the highest adult diabetic populations in sub-Saharan Africa. According to this study, patient attendance rates and medical admissions related to diabetes in major hospitals have been rising. This requires a shift in healthcare priorities and up-to-date data on the prevalence and related complications of diabetes in Ethiopia, to help plan and prioritize health programs. Similarly, the Ethiopia diabetes association (EDA) reported that currently diabetic patient attendance rates are alarmingly increasing than the past decayed and medical admissions in hospitals are also escalating. IDF (2013 and 2015) report also showed that in Ethiopia the total adult population is $49,483,000$ and the prevalence of diabetes in adults is $5.2 \%$ in the year 2017 and the total cases of diabetes in adults is 2,567,900. According to $\mathrm{WHO}$ estimation, there were about 800,000 people having diabetes in Ethiopia in 2000 and the number is expected to escalate to 1.8 million by the year 2030 (Admassu \& Gaym, 2009).

Such dreadful studies and reports are a great alarm for the government, health minister and all segments of the society to practice and make it culture of positive lifestyle in all the time before the problem aggravates, in line with this, the current study is therefore focused, to examine the effect of supervised vs. self-directed exercise and dietary practices on body weight and glycaemic control with individuals having type 2 diabetes.

\section{Methods}

\section{Subjects}

Subjects in this study were sixteen (16) voluntary type-2 male diabetic individuals whose aged were $54.0 \pm 4.5$ years old and with BMI between 25 to 29.9 $\mathrm{kg} / \mathrm{m}^{2}$. They did not also receive either supervised exercise training or dietary advice before.

\section{Inclusion Criteria: Health Screening}

Since the participants in this study were type-2 diabetics', before the commencement of the intervention or supervised training program, the ACSM's Guidelines for Exercise Testing and Prescription (Ehrman et al., 2018) recommendation were strictly applied as a framework criteria to avoid exposing habitually inactive individuals with known or occult cardiovascular disease (CVD) to the transiently heightened risks of unaccustomed type of exercise intensity, including sudden cardiac death (SCD) and acute myocardial infarction (AMI). It is also an important ethical and legal consideration and also mandatory procedure to apply the correct pre-exercise procedure in order to exercise training protocol to be effective and for the subjects' overall safety, as a result, subjects were first told to visit and consult thoroughly with their health care professionals (doctor) about the exercise training protocol and finally those subjects who didn't have contraindications for the exercise protocol and received approval or recommendation, started the exercise training protocol in a voluntary based. 


\section{Study Design}

This was experimental with retrospective cohort study design, in which health records of all subjects were obtained and collected from the patients themselves. In this study, all reasonable efforts were made to protect patients' private health information (e.g., medical history, test results, etc.,) and the study protocol was reviewed and approved by each subject ' medical doctors and consent was obtained from all participants. Subjects were classified randomly into two (2) groups, the names, experimental group (eight subjects living with type-2 diabetes and use oral antidiabetic drugs as per their physician advice and received supervised exercise (aerobic + resistance) and dietary advice by an exercise specialist and dietician respectively. The control group had similar characteristics of that of experimental groups except that, this group performed exercise by their own previous experience and also have their own dietary practices.

\section{Exercise Training Protocol and Dietary Practice}

The exercise training protocol was designed for 10 weeks which includes aerobic, resistance and flexibility exercise. Aerobic exercise (brisk walking, etc.): a minimum of 150 minutes/week of moderate-intensity $(50 \%$ to about $70 \%$ of individuals' maximum heart rate); Resistance exercise: at least 2-3 days/week of moderate (50\% of one-repetition maximum) and core body flexible exercise were also included. Generally, the participants were encouraged to engage in an exercise training program that was designed for diabetic individuals as per the (Ehrman et al., 2018; Feinglos \& Bethel, 2008) exercise training guidelines. The dietary practice of an experimental group was implemented through small groups and/or in one-on-one counseling by a clinical dietitian, whereas the control group subjects were followed by their usual eating Pattern.

\section{The Fasting Plasma Glucose Test (FPG)}

The FPG was performed after the subjects were fasted for at least 8 hours (Table 1). This fasting overnight plasma glucose test was conducted in the morning twice (pre and post) of the intervention in both groups. The level of glucose in the blood was measured based on the clinical laboratory protocol/methods by applying a drop of blood to a chemically treated, disposable 'test-strip', which was then inserted into an electronic blood glucose meter. The reaction between the test strip and the blood was detected by the meter and displayed in units of $\mathrm{mg} / \mathrm{dL}$ or $\mathrm{mmol} / \mathrm{L}$. American Diabetes Association (2019) stated that the FPG measures a person's blood sugar level after fasting or not eating anything for at least 8 hours. Normal fasting blood glucose is less than 100 milligrams per deciliter or $\mathrm{mg} / \mathrm{dL}$. A fasting plasma glucose greater than $100 \mathrm{mg} / \mathrm{dL}$ and less than $126 \mathrm{mg} / \mathrm{dL}$ implies that the person has an impaired fasting glucose level but may not have diabetes. A diagnosis of diabetes is made when the fasting blood glucose is greater than $126 \mathrm{mg} / \mathrm{dL}$ and when blood tests confirm abnormal results. These tests can be repeated on a subsequent day or by measuring glucose 2 hours after a meal.

Table 1

Diagnostic criteria for pre-diabetes and diabetes mellitus.

\begin{tabular}{lcc}
\hline & $\begin{array}{c}\text { Fasting Plasma } \\
\text { Glucose Test (FPG) }\end{array}$ & $\begin{array}{c}\text { 2-Hour Oral Glucose } \\
\text { Tolerance Test (OGTT) }\end{array}$ \\
\hline Normal & Below 100mg/dl & Below $140 \mathrm{mg} / \mathrm{dl}$ \\
Pre-diabetes & $100-125 \mathrm{mg} / \mathrm{dl}$ (IFG) & $140-199 \mathrm{mg} / \mathrm{dl}$ (IGT) \\
Diabetes & $126 \mathrm{mg} / \mathrm{dl}$ or above & $200 \mathrm{mg} / \mathrm{dl}$ or above \\
\hline
\end{tabular}

Source: American Diabetes Association. Diabetes Care 2007; 30; (Suppl.1): S4-41 


\section{Anthropometric Measurements}

In this study BMI, (a measurement of a person's weight-to-height ratio) was taken twice (pre and post-measurement). As stated by Gallagher \& LeRoith, (2012) body weight can be used as a surrogate measure for an individual's body fat mass. BMI is a measure of weight adjusted for height and is the commonest measure of body weight used in epidemiological studies as it is easy to measure accurately and correlates reasonably well with body fat measures but has also been proven to predict obesity-related conditions such as T2D. Through studies clear cut points for BMI have been defined, which are associated with risk for diabetes and other conditions. In adults a BMI between 25 and $29.9 \mathrm{~kg} \cdot \mathrm{m}^{-2}$; indicates being overweight while a BMI above $30 \mathrm{~kg} / \mathrm{m}^{2}$ indicates being obese.

\section{Statistical Analysis}

Baseline demographic values such as age and BMI are reported as mean $\pm \mathrm{SD}$. All outcome data are shown as mean \pm SEM. To assess for significance between participants taking supervised exercise and dietary advice and those not taking supervised exercise and dietary advice of each outcome from baseline up to 10 weeks intervention dependent $\mathrm{t}$ test was used. To determine whether there is statistical relationship between the baseline and post-intervention data of both groups, the correlation was used. Specifically Pearson's correlation was also used. In all cases, the statistical significance threshold was $p<0.05$.

\section{Results}

\section{Demographics and Fasting Plasma Glucose Test (FPG) at Baseline and Post results}

A total of 16 participants completed the intervention by October 05,2019 . Age $(54.0 \pm 4.5$ $\mathrm{yr})$ and BMI $(26.65 \pm 6.1 \mathrm{~kg} \cdot \mathrm{m}-2)$. The pre and post fasting plasma glucose test (FPG) results of the experimental and control group were displayed in (Table 2).

Table 2

Experimental \& Control Groups characteristics of different variables.

\begin{tabular}{|c|c|c|}
\hline Variables & Experimental Group $(\mathrm{n}=8)$ & Control Group $(\mathrm{n}=8)$ \\
\hline Age (yr) & $53.0 \pm 6.5$ & $55.0 \pm 2.5$ \\
\hline Height $\left(\mathrm{H}^{2}\right)$ & $170.5000^{\mathrm{a}} ; 170.500^{\mathrm{b}}$ & $167.13^{\mathrm{a}} ; 167.125^{\mathrm{b}}$ \\
\hline Weight (kg) & $78.00 \pm 3.6^{\mathrm{a}} ; 73.00 \pm 3.5^{\mathrm{b}}$ & $74.00 \pm 4.89^{\mathrm{a}} ; 73.255 .28^{\mathrm{b}}$ \\
\hline \multicolumn{3}{|l|}{ Paired Samples Statistics } \\
\hline$\tilde{\mathrm{x}}$ of $\mathrm{BMI}{ }^{\mathrm{a}} \& \mathrm{BMI}{ }^{\mathrm{b}}$ & $26.8500 \pm 0.96^{a} \& 25.1000 \pm 0.87 b$ & $26.45 \pm .66^{\mathrm{a}} ; 26.43 \pm .46^{\mathrm{b}}$ \\
\hline$\tilde{x}^{\text {of }}$ FPG $^{a} \&$ FPG $^{\mathrm{b}}$ & $205.37 \pm 48.72 \mathrm{a} ; 120.25 \pm 32.25 \mathrm{~b}$ & $212.75 \pm 51.95 ; 211.50 \pm 64.40$ \\
\hline \multicolumn{3}{|l|}{ Paired Samples Statistics } \\
\hline "r" of BMI a \& BMI b & 0.931 (corr.) & 0.252 (corr.) \\
\hline " $\alpha "$ of $\mathrm{BMI}{ }^{\mathrm{a}} \& \mathrm{BMI}{ }^{\mathrm{b}}$ & 0.001 (Sig) & 0.546 (Sig) \\
\hline "r" of FPGa \& FPG & 0.736 (corr.) & 0.488(corr.) \\
\hline " $\alpha "$ of $\mathrm{FPG}^{\mathrm{a}} \& \mathrm{FPG}^{\mathrm{b}}$ & 0.037(Sig & 0.220 (Sig) \\
\hline
\end{tabular}

Note: ${ }^{a}=$ the mean value of pre-test; ${ }^{b}=$ the mean value of post-test result; corr. $=$ correlation; Sig. $=$ Significant. FPG ${ }^{a}=$ Fasting Plasma Glucose Test at pre-test; FPG $=$ Fasting Plasma Glucose Test at post-test. $\tilde{x}=$ Mean; " $r$ " = correlation of sample of subjects; $\alpha$ = significance level. 


\section{Discussion}

Type 2 diabetes can often be prevented, mainly by eating a healthy and balanced diet, getting regular exercise, and keeping your weight within a healthy range. Weight loss can make your cells more sensitive to the effects of insulin, thereby helping to reduce blood sugar. If you are overweight, losing just 5 to 7 percent of your weight. If you already have diabetes, close monitoring of your blood sugar levels along with healthy eating and regular exercise can help you manage your condition and avoid serious complications. Even small changes in your lifestyle can produce big health benefits. Many people with type 2 diabetes can control their blood sugar with diet, exercise, and weight loss, but some need to take sugar-lowering medications or insulin injections (Metzger, 2006). The combined benefits of weight maintenance, physical activity, and healthy diet, however, have been demonstrated further by a recent series of diabetes prevention trials conducted in China, Finland, and the United States Knowler et al. 2002; Pan et al. 1997; Tuomilehto et al. 2001 (cited in Hawley \& Zierath 2008). The Finnish and American studies combined all lifestyle changes (reduced caloric intake, reduced fat intake, increased intake of fiber and whole grains, increased frequency and duration of moderate intensity physical activity) into a single multidisciplinary program Tuomilehto et al. 2001 (cited in Hawley \& Zierath 2008).

Similar to the aforementioned studies, in the present study, statistical significant (.001 and .037) were observed in all outcome measures for both body mass Index (BMI) and Fasting Plasma Glucose Test (FPG) respectively in the experimental groups. However, as it is displayed in table-2, with the same variables, the control group did not show any statistical significant ( $\mathrm{P}$ value of .546 and .220) were obtained for BMI and FPG respectively. The intervention of carefully designed and supervised exercise program such as aerobic, resistance and flexible exercise along with dietary advice and strict follow-ups brought successful weight loss and reduced FPG significantly for diabetes patients. Table- 2 also indicated the Pearson's correlation (also called Pearson's R) was used in linear regression of this study to measure how strong a relationship is between two variables (pre and post) test of BMI and FPG in both the experimental and control groups. The correlation coefficient of BMI was .931 and .252 for experimental and control group respectively, however, the FPG correlation in experimental and control group was obtained .736 and .488. This correlation value also indicates the impact of the degree of variables strength in both pre and post test results of BMI and FPG of both groups. Therefore, a higher correlation was obtained in the experimental groups. The strength of the correlation also indicates that participants who engaged in an exercise training program and received dietary close counselling dietary advice shows a better changes in weight loss and significantly manage their fasting blood glucose level than their counterparts.

Table 3

Paired difference value of experimental \& control group.

\begin{tabular}{|c|c|c|c|c|c|c|}
\hline \multirow{2}{*}{ Variables } & \multicolumn{3}{|c|}{ Paired } & \multirow{2}{*}{$\mathrm{t}$} & \multirow{2}{*}{ df } & \multirow{2}{*}{$\mathrm{p}$} \\
\hline & Mean & SD & SE & & & \\
\hline Pair 1. Exp.Group BMI ${ }^{\mathrm{a}}$ vs. BMI ${ }^{\mathrm{b}}$ & 1.75 & .35 & .12 & 14.122 & 7 & $.000^{*}$ \\
\hline Pair 1. Exp. Group FPG ${ }^{\mathrm{v}}{ }_{\mathrm{vs}}$. FPG ${ }^{\mathrm{b}}$ & 85.13 & 33.17 & 11.73 & 7.258 & 7 & $.000^{*}$ \\
\hline Pair 1. Cont. Group BMIa vs. BMI ${ }^{\mathrm{b}}$ & .0125 & .71 & .25 & .050 & 7 & .962 \\
\hline Pair 1. Cont. Group FPG ${ }^{a}$ vs. FPG ${ }^{b}$ & 1.25 & 59.86 & 21.17 & .59 & 7 & .955 \\
\hline
\end{tabular}

Note: $B M I^{a}=$ Body mass Index at baseline; $B M I^{b}=$ Body mass Index at Post-test. FPG ${ }^{a}=$ Fasting Plasma Glucose Test at baseline; FPG ${ }^{b}=$ Fasting Plasma Glucose Test at post-test. ${ }^{*} p<.05$ 
The data in Table 3 clearly demonstrate the mean paired value difference of BMI and FPG of both the experimental and control groups. The standard deviation, standard error, $95 \%$ confidence interval of the difference, $\mathrm{t}$-value, $\mathrm{df}$ and significant value of each variables are also clearly depicted.

Therefore, the mean difference value of BMI in the experimental group is 1.75 whereas .01250 value was obtained in the case of control group. The $\tilde{x}$ difference value of experimental groups of BMI was clearly seen at the $t$-value (14.122). This indicate that in the experimental group the exercise intervention and dietary advice brought great improvement than the control group.

Similarly the mean paired difference value of fasting plasma glucose test result in the experimental group is 85.13 while 1.25 value was obtained in the control group. This difference was also seen in the $t$-value which has 7.258 for experimental group and .059 for the control groups. This showed that the aerobic and resistance exercise intervention brought great improvement through moderate physical activity and dietary life style change than the control groups.

Metzger (2006) demonstrated that when combined with a healthy diet and weight loss, exercise is a safe and effective way to prevent diabetes. Exercise helps cells use the circulating blood sugar they need for energy production by making the cells more sensitive to the hormone insulin, even if you don't also lose weight. But a weight loss of just a few pounds can improve your insulin sensitivity even more. Aerobic and strength-training exercises are effective in preventing type 2 diabetes. Aerobic exercise makes cells more sensitive to insulin and, by burning calories and aiding weight loss, can help keep blood glucose levels from rising. Strength training decreases body fat by raising metabolism and building muscle, which burns more calories than fat. People at risk for diabetes is that strength training can help make your cells more sensitive to insulin and enable muscle cells to absorb sugar from the blood more efficiently. Resistance training helps you lose body fat and rebuild muscle.
Similar to the present study, a recent another meta-analysis of 10 prospective cohort studies showed that participating regularly in moderateintensity physical activity or in a brisk walking regimen reduced diabetes incidence by $30 \%$ (Jeon et al., 2007). Most observational studies suggest that energy expenditure is the most important attribute of physical activity in terms of lowering diabetes risk and suggest that public health recommendations should encourage regular, moderate-intensity physical activity as opposed to high-intensity exercise (Hawley \& Zierath, 2008). Exercise training enhances sensitivity to insulin and also increases oxidation of triglycerides within the muscle.

Another previous study indicated that remarkably, 9 in 10 cases of type 2 diabetes could be prevented by a simple lifestyle modification that some people have erroneously categorized as intense. For example, moderate physical activity, such as brisk walking for $2.5 \mathrm{~h} / \mathrm{wk}$. (22 $\mathrm{min} / \mathrm{d}$ ), is capable of reducing the risk of overt diabetes by at least $66 \%$ in individuals with prediabetes, as compared to walking for less than $1 \mathrm{~h} / \mathrm{wk}$. Apart from this, in many ways, physical activity improves quality of life. Despite the billions of dollars flowing to pharmaceutical companies, the most efficacious preventative antidiabetic drug is still physical activity. Health care professionals as well as the general public realize and implement physical activity as the most effective exit strategy against type 2 diabetes (Hawley \& Zierath, 2008).

Various studies also reported that dietary elements have been identified as potential modifiable risk factors for diabetes. Greater intakes of saturated fat, sugar-sweetened soft drinks, and fast food have been associated with increased risk for diabetes. A high proportion of polyunsaturated fats in the diet and higher intake of fish oils, wholegrain and cereal fibers, and dairy have each been associated with a reduced risk of diabetes. Similarly, higher levels of caffeine and coffee intake and moderate alcohol consumption decreased the diabetes risk in prospective population-based studies $\mathrm{Hu}$, van Dam, \& Liu (cited in Hawley \& Zierath, 2008). Similarly the current study advice subjects to avoid many of the diets which 
contributed as potential modifiable risk factors to the experimental subjects and focused on polyunsaturated fats, whole-grain, and cereals.

\section{Conclusion}

The present finding revealed that both the experimental \& control groups were able to manage their blood glucose level; however; a significant glycemic control and body weight reduction was observed only in the experimental group. Therefore, to control the burden of the inactivity-related diseases specifically diabetes, diabetic patients and even the public in general to take up moderate regular exercise and use it as their treatment of choice. Thus, exercise should be incorporated as a part of the everyday life. However, for reliable and sustainable glycaemic control, individuals who are living with type-2 diabetes should get close guidance by exercise specialist and dietitians in addition to their usual medical treatments/ flow-ups/.

In association to this finding, the Ethiopia national diabetes association explained that diabetes prevalence will be projected to increase alarmingly unless the people lifestyle is changed and all pertinent individuals and organizations discharge their duties and responsibilities properly. A sedentary lifestyle-for both healthy and diseased people is detrimental and it is a universally accepted standpoint. Similar to the present study, many other intervention studies have demonstrated that currently, there are several pharmacological treatment options for patients with type 2 diabetes, depending on the stage and progression of their symptoms however; none is without adverse side effects, and the consequences of their long-term use are largely unknown.

Therefore, in conclusion, all individuals with diabetes or prediabetes should be encouraged to be regularly physically active, including more daily physical movement and structured exercise, to improve their health and longevity. For example, in this regard, the federal government of Americans has established new guidelines for exercise. The guidelines recommend at least 30 minutes of moderate-intensity physical activity every day to reduce the risk of chronic diseases such as type 2 diabetes and heart disease. The guidelines also call for 60 minutes of moderate to vigorous physical activity most days to prevent weight gain, and 60 to 90 minutes of daily physical activity to lose weight (Metzger, 2006). Understanding the existing and upcoming health problem caused by T2D, the Ethiopia government, healthcare sectors and other stakeholders should be actively involved to establish strong and sustainable health policy initiatives related to education programs, awareness creation of the risk of diabetes to the whole population and to empower people to improve in maintaining a healthy weight by exercising regularly and develop healthy eating habits to prevent $\mathrm{T} 2 \mathrm{D}$ and its complications.

\section{References}

Abebe N, Kebede T, Addise D. Diabetes in Ethiopia 2000-2016 prevalence and related acute and chronic complications; a systematic review. African Journal of Diabetes Medicine, 2017; 25(2): 7-11.

Admassu G, Gaym A.Outcome of pregnancy complicated by diabetes at Tikur Anbessa Hospital, Addis Ababa Ethiopia ĐA five-year review. Ethiopian Journal of Reproductive Health, 2009; 3(3): 34-40.

American Diabetes Association. National Institute of Diabetes and Digestive and Kidney Diseases. Xylitol.org. News release, FDA, 2019.

Ehrman RD JK, Liguori G, Magal M. ACSM's guidelines for exercise testing and prescription. 10 $0^{\text {th }}$ Edition, Philadelphia: Wolters Kluwer, 2018.

Feinglos MN, Bethel MA. Type 2 Diabetes Mellitus: An evidence-based approach to practical management. USA: Human Press, 2008.

Gallagher EJ, LeRoith D. Prevention of Type 2 Diabetes from science to therapy. New York: Springer, 2012.

Hall V, Thomsen R, Henriksen O, Lohse N. Diabetes in Sub Saharan Africa 1999 \pm 011: Epidemiology and public health implications. A systematic review. BMC Public Health, 2011; 11(564).

Hawley JA, Zierath JR. Physical activity and type 2 diabetes: Therapeutic effects and mechanisms of action. United Sated of America: Human Kinetics, 2008. 
International Diabetes Federation (2013). IDF Diabetes Atlas. 6th ed. Brussels, Belgium: IDF, http://www.idf.org/diabetesatlas Accessed May 11 2019.

International Diabetes Federation. Diabetes Atlas. IDF. 7th edition, 2015.

Jeon CY, Lokken R, Hu FB, van Dam RM. Physical activity of moderate intensity and risk of type 2 diabetes. A systematic review. Diabetes Care, 2007; 30: 744-752.
Metzger BE. American medical association guide to living with Diabetes. New Jersey: John Wiley \& Sons, Inc., 2006.

Potteiger JA. ACSM's introduction to exercise science. $3^{\text {rd }}$ Edition, Philadelphia: Wolters Kluwer, 2018.

Shaw JE, Sicree RA, Zimmet PZ. Global estimates of the prevalence of diabetes for 2010 and 2030. Diabetes Research and Clinical Practice, 2010; 87: 4-14. 